\title{
Coupling of Substructures for Dynamic Analyses
}

\author{
Roy R. Cratg JR.* \\ The University of Texas at Austin, Austin, Texas \\ AND \\ Mervyn C. C. Bampton $\dagger$ \\ The Boeing Company, Seattle, Wash.
}

\begin{abstract}
A method for treating a complex structure as an assemblage of distinct regions, or substructures, is presented. Using basic mass and stiffness matrices for the substructures, together with conditions of geometrical compatibility along substructure boundaries, the method employs two forms of generalized coordinates. Boundary generalized coordinates give displacements and rotations of points along substructure boundaries and are related to the displacement modes of the substructures known as "constraint modes." All constraint modes are generated by matrix operations from substructure input data. Substructure normal-mode generalized coordinates are related to free vibration modes of the substructures relative to completely restrained boundaries. The definition of substructure modes and the requirement of compatibility along substructure boundaries lead to coordinate transformation matrices that are employed in obtaining system mass and stiffness matrices from the mass and stiffness matrices of the substructures. Provision is made, through a RayleighRitz procedure, for reducing the total number of degrees of freedom of a structure while retaining accurate description of its dynamic behavior. Substructure boundaries may have any degree of redundancy. An example is presented giving a free vibration analysis of a structure having a highly indeterminate substructure boundary.
\end{abstract}

\section{Nomenclature}

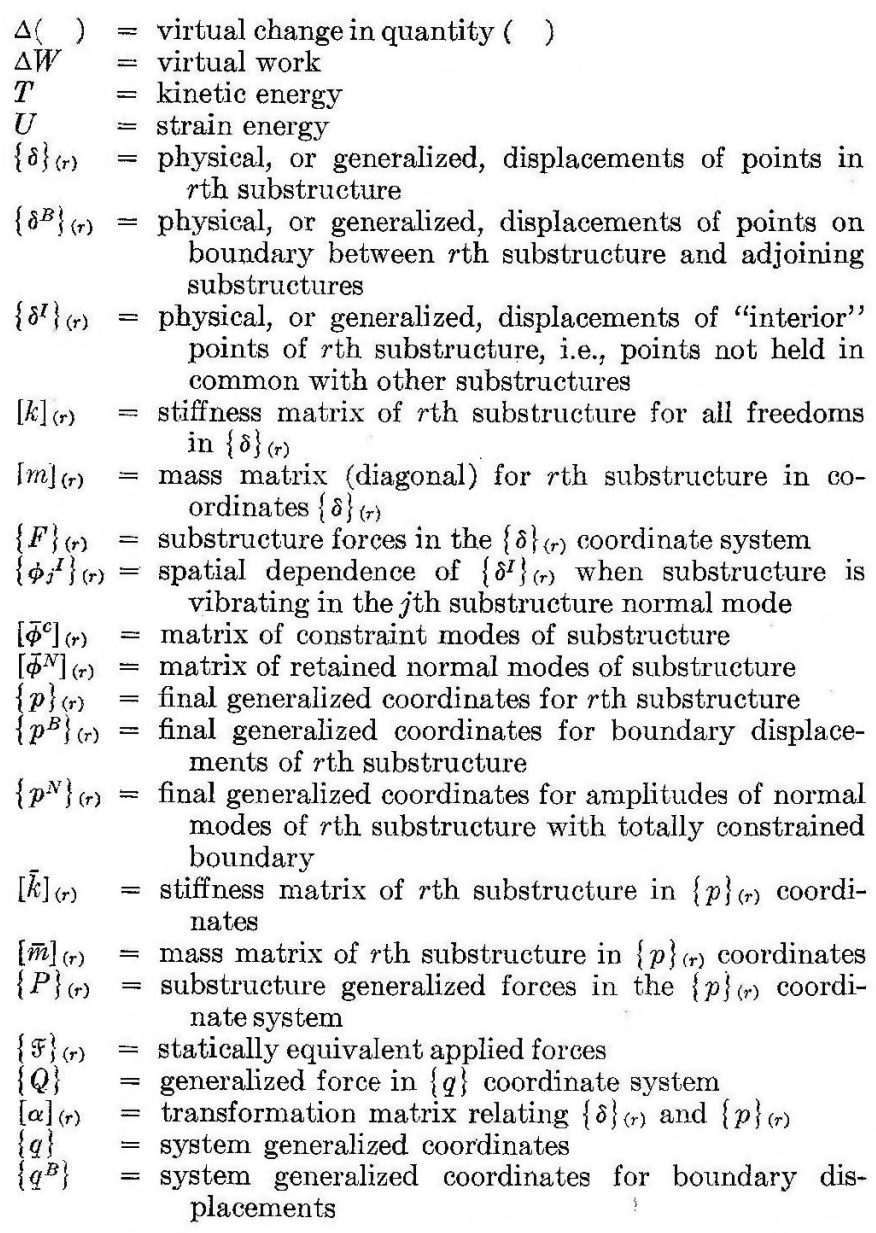

* Associate Professor of Aerospace Engineering. Member AIAA.

† Dynamics Research Engineer, Commercial Airplane Division.
$\left\{q^{N}\right\}=$ system generalized coordinates related to normal modes of constrained substructures

$[K]=$ stiffness matrix for total structure in $\{q\}$ generalized coordinate system

$[M]=$ mass matrix in $\{q\}$ system coordinates

$\{\delta\} \equiv\left\{\begin{array}{c}\delta_{(1)} \\ \delta_{(2)} \\ \cdot \\ \cdot \\ \delta_{(s)}\end{array}\right\}=$ collection of substructure vectors, $\{\delta\}_{(r)}$

$\{p\} \equiv\left\{\begin{array}{c}p_{(1)} \\ p_{(2)} \\ \cdot \\ \cdot \\ p_{(s)}\end{array}\right\}=$ collection of substructure vectors, $\{p\}_{(r)}$

$[\beta]=$ transformation matrix relating $\{p\}$ and $\{q\}$

\section{Introduction}

$\checkmark$ THE fundamental problems in the analysis of structural systems subjected to time-dependent forces are the determination of the displacement response of the system and the internal stresses. One approach to the solution of these problems is to idealize the system as an assemblage of discrete structural elements, thereby obtaining sets of equations which are conveniently treated by the operations of matrix algebra. In order to obtain the detailed displacement and stress information required in the analysis of a complex structure, it may be necessary to divide the structure into a very large number of elements, thereby introducing matrices of large order.

To retain such large matrices and analyze the structure as a whole may require computer facilities of greater capacity than those that are available. At any rate, it fails to take into account the procedures usually employed in the design or modification of complex structures whereby major structural components, or substructures, are often designed by different engineering groups or at different times. It is desirable, therefore, to use a substructure approach so that such designs and modifications may proceed as independently 


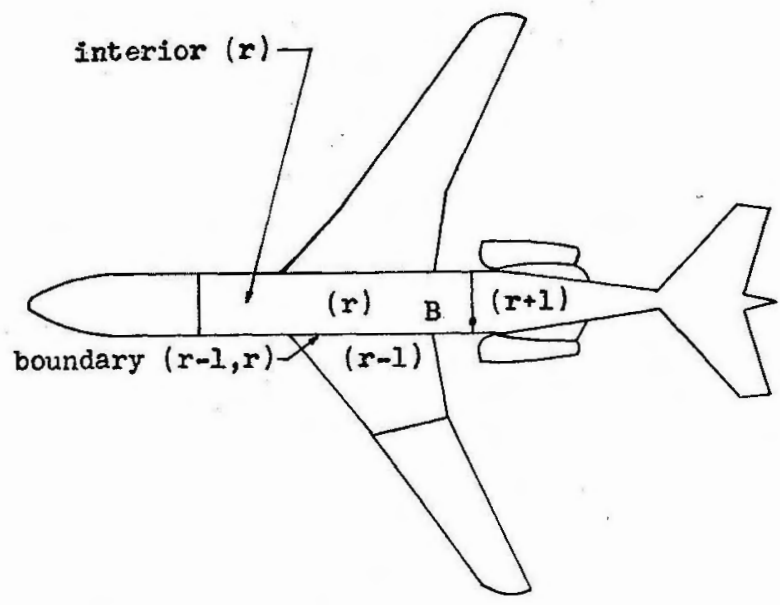

Fig. 1 Typical substructure arrangement.

as possible with due consideration being given to the final coupling of substructures to form the complete structure. At the same time, the order of the final matrices may be reduced by incorporating a Rayleigh-Ritz procedure into the substructure method.

The hierarchy of structures to be considered in this paper consists of the structural system, the substructure, and the element. It is the process of dividing the system into substructures and coupling these again to form the system that is treated here. It is assumed that analysis of the individual substructures by discrete element idealization has been carried out to obtain the necessary substructure stiffness matrices and mass matrices.

In the present discussion, a lumped-mass formulation is used, although other procedures for obtaining substructure mass matrices could be employed. ${ }^{1}$ The displacement method is employed in the final coupling of substructures. Substructure methods that rely upon the displacement method, i.e., upon geometric compatibility along substructure boundaries, for coupling of the substructures have been employed in analysis of both static ${ }^{2,3}$ and dynamic ${ }^{4,5}$ behavior of structures. Each of the latter employs a Rayleigh-Ritz procedure to reduce the order of the final system mass and stiffness matrices. The "branch-modes" technique discussed by Gladwell ${ }^{6}$ has the advantage that, for a special choice of branches or substructures, the system stiffness matrix is diagonal and is easily formed along with the normalmode analysis of the branches. For other divisions of the system, the branch-modes method requires formation of a stiffness matrix in the original system coordinates, with subsequent reduction to final system coordinates by the Rayleigh-Ritz method.

The two procedures of Gladwell ${ }^{6}$ for choosing the branches are: 1) "by demanding that one of the components vibrates with distortion while the other vibrates as a rigid body attached to the distorting component;" 2) "by demanding that one of the components vibrates with distortion while the other remains fixed." In either case it may be seen that the boundary between two substructures is assumed to be rigid, a restriction that cannot be accepted for cases of highly redundant boundaries between substructures. The present paper may be considered as a generalization of branch-modes procedures to structures with such redundant substructure boundaries.

Hurty ${ }^{4,5}$ has developed a procedure for the analysis of structural systems in which the displacement behavior of each substructure is described by a set of generalized coordinates consisting of the following three types: 1) rigidbody coordinates, 2) constraint coordinates, and 3) normalmode coordinates. Since the present paper differs from Refs. 4 and 5 principally in the selection of generalized coordinates for substructures, the significance of the generalized coordinates used by Hurty requires further elucidation here.
A substructure is considered to be composed of interior degrees of freedom and boundary degrees of freedom. If the number of boundary freedoms is greater than the number of rigid-body freedoms of the substructure, the former are separated by Hurty into "statically determinate constraints" and "redundant constraints." Displacements of a substructure are defined in terms of generalized coordinates that are related to specified sets of normalized displacement functions. The modes are considered, by Hurty, ${ }^{3}$ in three categories as follows:

First are rigid-body displacements in which the component (substructure) is displaced without deformation. If no fixed external constraints are imposed on the component, it will have six degrees of freedom as a rigid body; hence there may be as many is six rigid-body modes for the component. This number will be reduced if external constraints are present. In the second category are modes that will exist only if the system of constraints on the component is indeterminate. These modes are defined by producing a unit displacement on each redundant constraint in turn, with all other constraints fixed. For brevity these modes are ealled "constraint modes," and they are equal in number to the number of redundant constraints. The third category includes modes that define displacements relative to the constraint system. They may be, for example, the normal modes of vibration of the component with all constraints fixed.

As rigid-body modes Hurty ${ }^{5}$ suggests the use of rigid-body translations and small rotations. Hence, the displacements of the determinate constraints must be expressed in terms of these rigid-body motions, since it is the former that will appear in the equations expressing compatibility of displacements along substructure boundaries. The total number of generalized coordinates to be used in describing a structural system may be reduced, in the Hurty method, by using only a portion of the total possible normal modes of the constrained substructures in describing the displacement of interior points relative to the constraints.

A number of basic simplifications occur if all of the boundary freedoms are treated alike, rather than being separated into determinate and indeterminate constraints. Since, for a highly redundant boundary connection it is not at all clear which freedoms should be treated as "statically determinate" and which as "redundant," it is highly advantageous to have a method that does not require the engineer to make such distinctions. In addition, it will be shown that the formulation of rigid-body modes and the establishment of equations relating rigid-body generalized coordinates to statically determinate constraint displacements is unnecessary when all boundary freedoms are treated alike. Therefore, in the present paper all boundary displacements will be treated in the same manner that Hurty uses for "redundant constraints." To treat the displacements of substructures as being composed of "constraint modes" and normal modes does not preclude the possibility of analyzing, by the present substructure procedures, a structural system that has rigidbody freedom, provided that constraint modes are defined for all boundary freedoms.

In the present analysis the constraint modes of a substructure are defined by producing a unit displacement of each boundary freedom in turn, with all other boundary freedoms fixed and with all interior freedoms unconstrained. The normal modes of a substructure describe the motion of interior freedoms relative to the fixed boundaries in terms of the normal modes of free vibration of the substructure with all boundary freedoms fixed. Equations of compatibility relate the displacements of adjoining substructures. The compatibility equations are used to determine a set of system boundary generalized coordinates which is equal in number to the total number of substructure boundary freedoms minus the number of compatibility equations. The final set of system generalized coordinates consists, then, of the system boundary generalized coordinates plus generalized coordinates for the substructure normal modes of all of the substructures. Lagrange's equations are used to derive the 
equations of motion for the system. The system stiffness matrix $[K]$ is found to have a particularly simple diagonalbanded form. In this respect, the present analysis is the logical mathematical extension of the branch-modes method to systems having highly redundant substructure boundaries.

\section{Substructure Constraint Modes and Normal Modes; Substrueture Mass Matrix and Stiffness Matrix}

Figure 1 shows a typical $r$ th substructure and its boundaries with substructures $(r-1)$ and $(r+1)$. Each substructure is connected to one or more other substructures. For the present analysis it is necessary to isolate the $r$ th substructure from all connecting substructures when the boundary freedoms are totally constrained. This may necessitate incorporation of some dummy boundary freedoms. This is illustrated by Fig. 2 , in which a cantilever plate is divided into two substructures. The original coordinates of the problem are taken to be the transverse displacements $\{\xi\}$ of the nodal grid points. In order that the boundary freedoms will allow complete isolation of the substructures it is necessary to introduce dummy boundary freedoms, in this case the rotations at the boundary nodal points as shown.

It is assumed that the stiffness matrix $[k]_{(r)}$ is available. Partitions of $[k]_{(r)}$ are formed as shown in Eq. (1):

$$
\left\{\begin{array}{c}
F^{B} \\
\hdashline F^{I}
\end{array}\right\}_{(r)}=\left[\begin{array}{c:c}
k^{B B} & k^{B I} \\
\hdashline k^{I B} & k^{I I}
\end{array}\right]_{(r)}\left\{\frac{\delta^{B}}{\delta^{I}}\right\}_{(r)}
$$

where superscripts $B$ and $I$ refer to boundary and interior, respectively. The coordinates $\left\{\delta^{B}\right\}_{(r)}$ are physical displacements of boundary points. In the present analysis $\left\{\delta^{I}\right\}_{(r)}$ are the physical displacements of interior points, although for other substructure idealizations, e.g. Ref. $1,\left\{\delta^{I}\right\}_{(r)}$ may be any other set of specified generalized coordinates describing the deformation of the interior of the substructure.

Constraint modes will be defined as the mode shapes of the interior freedoms due to successive unit displacement of boundary points, all other boundary points being totally constrained. To determine constraint modes the forces at all interior freedoms are set equal to zero. Equation (1) gives

$$
\{0\}=\left[k^{I B}\right]\left\{\delta^{B}\right\}+\left[k^{I I}\right]\left\{\delta^{I}\right\}
$$

or

$$
\left\{\delta^{I}\right\}=-\left[k^{I I}\right]^{-1}\left[k^{I B}\right]\left\{\delta^{B}\right\} \equiv\left[\bar{\phi}^{C}\right]\left\{\delta^{B}\right\}
$$

The subscript $(r)$ will be omitted when no confusion arises thereby. The matrix $\left[\bar{\phi}^{c}\right]$ is the desired matrix of constraint modes.

The matrix partition $\left[k^{I I}\right]$ gives the forces at interior freedoms due, successively, to unit displacement of interior freedoms with all boundary points totally constrained. Let the diagonal mass matrix, obtained from a lumped-mass formulation, be partitioned consistent with Eq. (1):

$$
\lceil m]=\left[\begin{array}{c:c}
m^{B B} & 0 \\
\hdashline 0 & m^{I I}
\end{array}\right]
$$

The substructure normal modes will be defined as the normal modes of the substructure with totally constrained boundary. These are obtained from the equations

$$
\left\{\delta^{I}\right\}=\left\{\phi^{I}\right\} e^{i \omega t} \quad \omega^{2}\left[m^{I I}\right]\left\{\phi^{I}\right\}=\left[k^{I I}\right]\left\{\phi^{I}\right\}
$$

The eigenvectors of Eq. (4) are the normal modes of the constrained substructure. These eigenvectors form the respective columns of the matrix $\left[\phi^{N}\right]$.

A fundamental assumption of the present analysis is that each $\left\{\delta^{I}\right\}_{(r)}$ contains a reasonably large number of elements, i.e., interior degrees of freedom, and that the contribution of the substructure normal modes to the displacement of these interior freedoms can be approximated by a significantly smaller set of coordinates $\left\{p^{N}\right\}_{(r)}$. Let $\left[\phi^{N}\right]_{(r)}$ be partitioned according to

$$
\left.\left[\phi^{N}\right]_{(r)}=\left[\bar{\phi}^{N}\right\rangle^{N}\right]_{(r)}
$$

where the modes to be retained are collected in $\left[\bar{\phi}^{N}\right]_{(r)}$. Let the elements of $\left\{p^{N}\right\}_{(r)}$ be the amplitudes of the respective modal vectors contained in $\left[\bar{\phi}^{N}\right]$, and let

$$
\{p\}_{(r)}=\left\{\begin{array}{c}
p^{B} \\
p^{N}
\end{array}\right\}_{(r)}
$$

The coordinate transformation relating the substructure final coordinates to the substructure initial coordinates is, then,

$$
\{\delta\}_{(r)}=[\alpha]_{(r)}\{p\}_{(r)}
$$

where

$$
[\alpha]_{(r)}=\left[\begin{array}{c:c}
I & 0 \\
\hdashline \bar{\phi}^{C} & \bar{\phi}^{N}
\end{array}\right]_{(r)}
$$

Fig. 2 Original substructure coordinates and substructure boundary displacement coordinates.

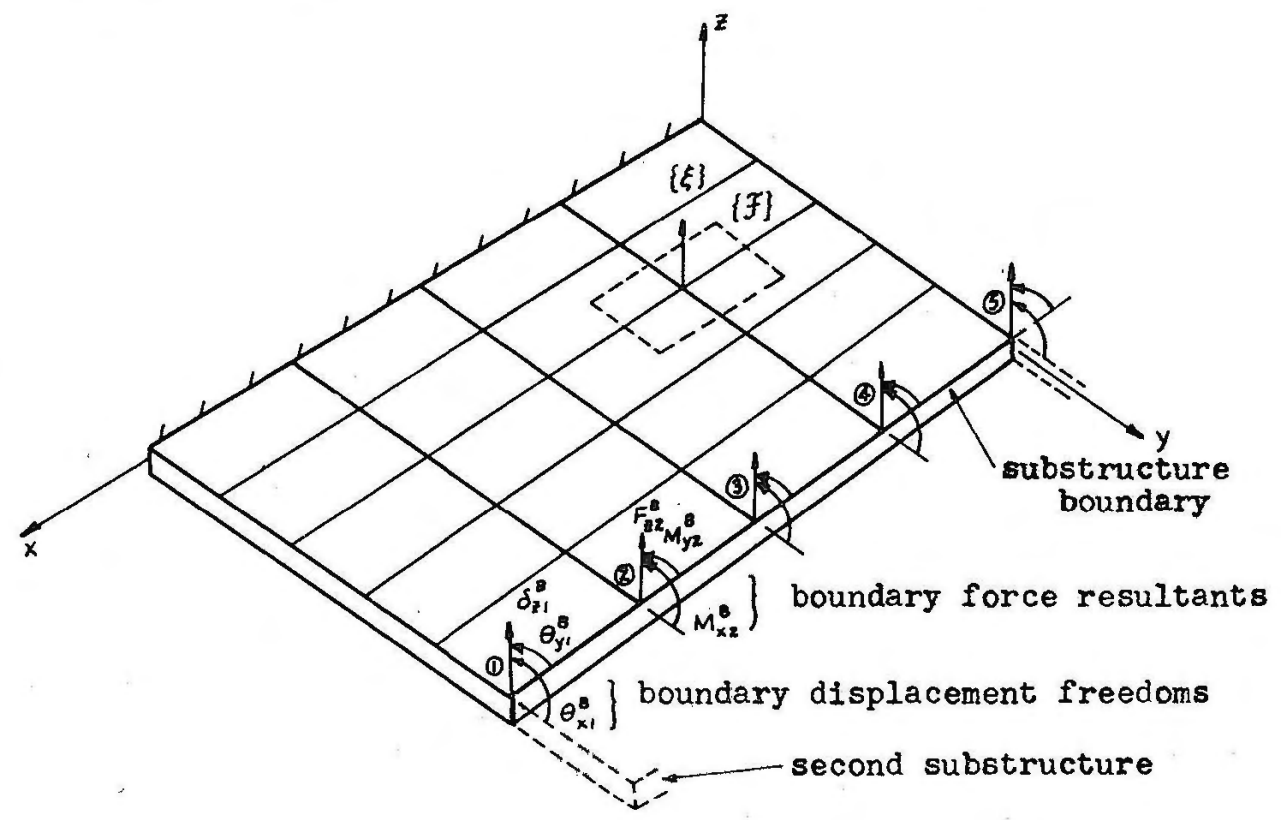


The unit matrix occurring in $[\alpha]_{(r)}$ implies that

$$
\left\{p^{B}\right\}_{(r)} \equiv\left\{\delta^{B}\right\}_{(r)}
$$

The stiffness matrix in the final set of substructure generalized coordinates $\{p\}_{(r)}$ is

$$
[\bar{k}]=[\alpha]^{\prime}[k][\alpha]=\left[\begin{array}{c:c}
\bar{k}^{B B} & 0 \\
\hdashline 0 & \bar{k}^{N N}
\end{array}\right]
$$

The matrices $\left[\bar{k}^{B N}\right]$ and $\left[\bar{k}^{N B}\right]$ are null due to the way in which $[\alpha]$ has been defined in Eq. (8). Furthermore,

$$
\left[\bar{k}^{B B}\right]=\left[k^{B B}\right]+\left[k^{B I}\right]\left[\bar{\phi}^{c}\right]
$$

Finally,

$$
\left[\bar{k}^{N N}\right\rfloor=\left[\bar{\phi}^{N}\right]^{\prime}\left[k^{I I}\right]\left[\bar{\phi}^{N}\right]
$$

which is diagonal due to the orthogonality of the substructure normal modes. The nullity of the off-diagonal submatrices is fundamental to the simplicity of the final system stiffness matrix.

The mass matrix for the substructure is given by

$$
[\bar{m}]=[\alpha]^{\prime}[m][\alpha]=\left[\begin{array}{c:c}
\bar{m}^{B B} & \bar{m}^{B N} \\
\hdashline \bar{m}^{N B} & \bar{m}^{N N}
\end{array}\right]
$$

The constituent submatrices are

$$
\begin{aligned}
{\left[\bar{m}^{B B}\right] } & =\left[m^{B B}\right]+\left[\bar{\phi}^{C}\right]^{\prime}\left[m^{I I}\right]\left[\bar{\phi}^{C}\right] \\
{\left[\bar{m}^{B N}\right] } & =\left[\bar{m}^{N B}\right]^{\prime}=\left[\bar{\phi}^{C}\right]^{\prime}\left[m^{I I}\right]\left[\bar{\phi}^{N}\right] \\
{\left[\bar{m}^{N N}\right] } & =\left[\bar{\phi}^{N}\right]^{\prime}\left[m^{I I}\right]\left[\bar{\phi}^{N}\right]
\end{aligned}
$$

The matrix $\left[\bar{m}^{N N}\right]$ is diagonal due to the orthogonality properties of the substructure normal modes. The elements of $\left[\bar{m}^{N N}\right]$ are related to corresponding elements of $\left[\bar{k}^{N N}\right]$ by the equations

$$
\bar{k}_{j} N N=\omega_{j}^{2} \bar{m}_{j}^{N N}
$$

where $\omega_{j}$ is the natural frequency of the $j$ th substructure normal mode.

Let $\Delta W$ be the virtual work done by forces $\{F\}$ in an arbitrary virtual displacement $\{\Delta \delta\}$. The generalized forces in the $\{p\}$ coordinate system are, by definition, those forces that do an amount of virtual work equal to $\Delta W$ when the $\{p\}$ coordinates undergo a virtual displacement consistent with $\{\Delta \delta\}$ :

$$
\Delta W=\{P\}^{\prime}\{\Delta p\}=\{F\}^{\prime}\{\Delta \delta\}
$$

Substitution of Eq. (7) into Eq. (16) gives

$$
\{P\}^{\prime}\{\Delta p\}=\{F\}^{\prime}[\alpha]\{\Delta p\}
$$

Since $\{\Delta p\}$ is arbitrary,

$$
\{P\}^{\prime}=\{F\}^{\prime}[\alpha]
$$

or

$$
\{P\}=[\alpha]^{\prime}\{F\}
$$

\section{Geometrical Compatibility of Substructure Boundaries}

Figure 1 illustrates typical substructure arrangements for an airplane. As indicated previously, each substructure is connected to one or more other substructures along boundaries that can be totally constrained. Thus, if points along the boundary of a given substructure are totally constrained, forces applied at interior points cause reactions only along the boundary of the given substructure. On the other hand, if a given boundary point is subjected to a unit displacement while all other points are totally constrained, forces will be produced only at the interior points of the substructures adjacent to the displaced boundary point and along the boundaries of these substructures. For example, a unit displacement of point $B$ in Fig. 1 will cause forces only within, and along the boundaries of, substructures $(r)$ and $(r+1)$.

Compatibility conditions are needed to insure that the displacements on the boundary of substructure $(r)$ match those of its adjoining substructures. No compatibility conditions exist among the normal mode coordinates $\left\{p^{N}\right\}(r)$ of the various substructures. Therefore, let

$$
\left\{q^{N}\right\}_{(r)} \equiv\left\{p^{N}\right\}_{(r)}
$$

The compatibility equations relating all boundary displacements may be written, symbolically, in the form

$$
\left[A_{d !}^{i} A_{\theta}\right]\left\{\frac{p_{d} B}{p_{g} B}\right\}=\{0\}
$$

where the $\left\{p_{t}^{B}\right\}$ are dependent variables and $\left\{p_{g}{ }^{B}\right\}$ are independent, or generalized, coordinates. $\left[A_{d}\right]$ is a square matrix. Let

$$
\left\{p_{g}^{B}\right\} \equiv\left\{q^{B}\right\}
$$

Then, from Eq. (21)

$$
\left\{\frac{p_{d}{ }^{B}}{\bar{p}_{o}{ }^{B}}\right\}=\left[\frac{-\left[A_{d}\right]^{-1}\left[A_{g}\right]}{[I]}\right]\left\{q^{B}\right\}
$$

Since

$$
\left\{p^{B}\right\} \equiv\left\{\begin{array}{c}
p_{(1)}^{B} \\
\cdot \\
\cdot \\
\cdot \\
p_{(s)}^{B}
\end{array}\right\}
$$

is not, in general, arranged according to the partitions shown in Eq. (21), the transformation matrix in Eq. (23) expresses in essence, although possibly not in exact form, the relationship between $\left\{p^{\mathrm{B}}\right\}$ and $\left\{q^{B}\right\}$.

The general form of the compatibility equation may be written

$$
[B]\left\{p^{B}\right\}=\{0\}
$$

and the general form of the coordinate transformation is then

$$
\left\{\begin{array}{c}
p_{(1)^{B}} \\
\cdot \\
\cdot \\
p_{(s)^{B}}
\end{array}\right\}=\left[\begin{array}{c}
\beta_{(1)^{B}} \\
\cdot \\
\cdot \\
\beta_{(s)^{B}}
\end{array}\right]\left\{q^{B}\right\}
$$

Finally, the general form of the coordinate transformation from $\{p\}$ to $\{q\}$ is

$$
\{p\}=[\beta]\{q\}
$$

or

$$
\{p\}_{(r)}=[\beta]_{(r)}\{q\}
$$

Let

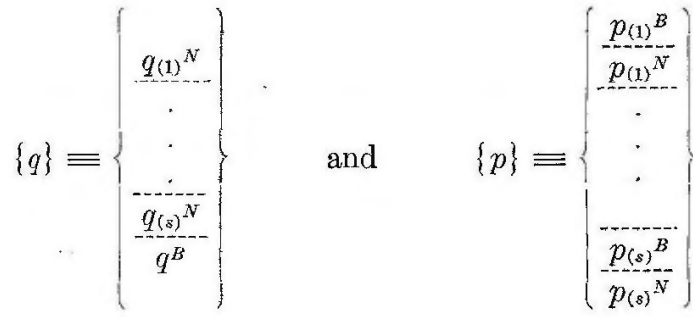


Then $[\beta]$ has the form

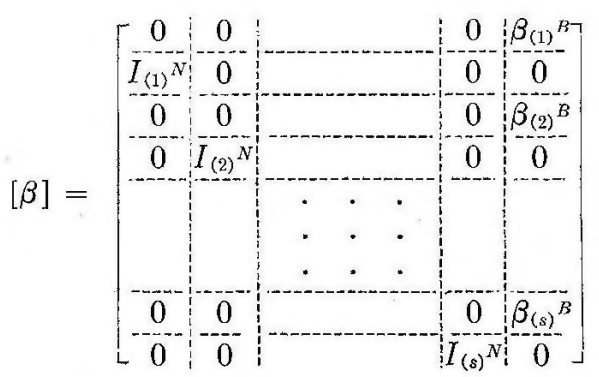

and $[\beta]_{(r)}$ has the form

$$
[\beta]_{(r)}=\left[\begin{array}{l|l|l|l}
\therefore & 0 & \ldots & \beta_{(r)}{ }^{B} \\
\hdashline \cdot \cdot & I_{(r)^{N}} & \cdots & 0
\end{array}\right]
$$

$(r)$

$(s)$

\section{System Equations of Motion}

The coordinate transformation of Eqs. (27) and (28) allows the mass and stiffness matrices for the system to be obtained. These may then be used in deriving the equations of motion through the use of Lagrange's equations. The system mass matrix and system stiffness matrix are obtained by summing, respectively, the kinetic energies of the substructures and the strain energies of the substructures.

Let

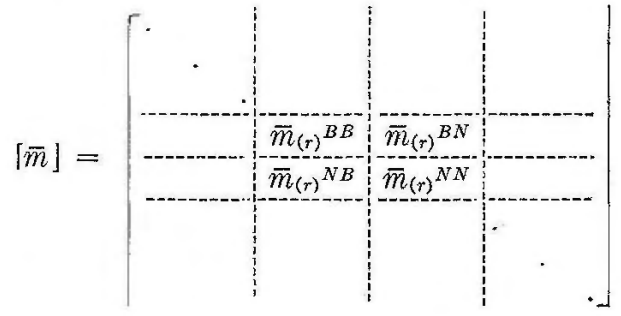

$[\bar{m}]$ is a diagonal-banded matrix with the substructure mass matrices along the diagonal. Because of the diagonal character of $\lceil\bar{m}\rfloor$, the following relationship holds:

$$
[M]=\sum_{r}[\beta]_{(r)}{ }^{\prime}[\bar{m}]_{(r)}[\beta]_{(r)}=[\beta]^{\prime}[\bar{m}][\beta]
$$

where $[M]$ is the final system mass matrix. Furthermore, let

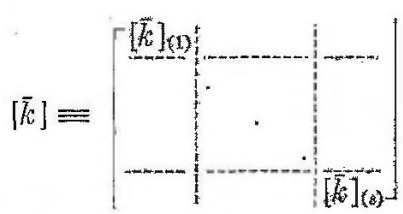

Then, due to the diagonal character of $[\bar{k}]$,

$$
\left.[K]=\sum_{r} \cdot[\beta]_{(r)}{ }^{\prime}[\bar{k}]_{(r)}[\beta]_{(r)}=[\beta]^{\prime} \mid \bar{k}\right][\beta]
$$

where $[K]$ is the system stiffness matrix in $\{q\}$ coordinates. When Eq. (31) is combined with Eq. (35), the final stiffness matrix is found to exhibit the diagonal character shown below:

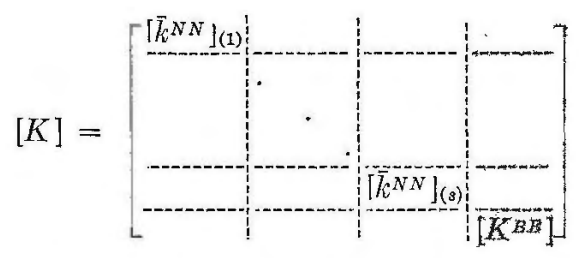

where

$$
\left[K^{B B}\right]=\sum_{r}\left[\beta^{B}\right]_{(r)}^{\prime}\left[\bar{k}^{B B}\right]_{(r)}\left[\beta^{B}\right]_{(r)}
$$

When $[K]$ is formed by Eqs. (36) and (37), the handling of large numbers of zeros, as required by Eq. (35), is avoided. The system mass matrix may likewise be found in a more efficient manner than that given in Eq. (33). When Eq. (31) is combined with Eq. (33), $[M]$ is found to have the form

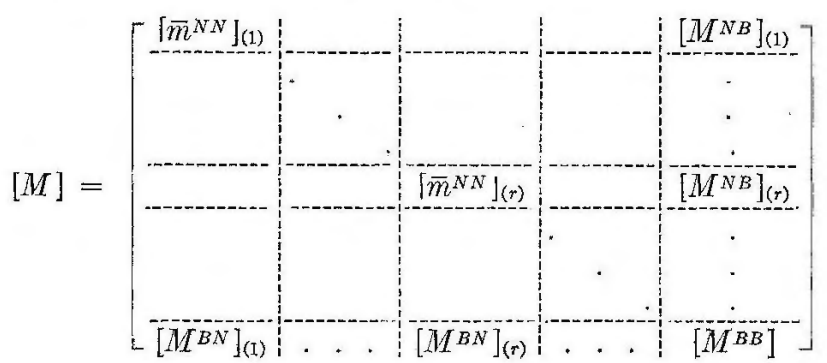

where

$$
\left[M^{N B}\right]_{(r)}=\left[M^{B N}\right]_{(r)}{ }^{\prime}=\left[\bar{m}^{N B}\right]_{(r)}\left[\beta^{B}\right]_{(r)}
$$

and

$$
\left[M^{B B}\right]=\sum_{r}\left[\beta^{B}\right]_{(r)}{ }^{\prime}\left[\bar{m}^{B E}\right]_{(r)}\left[\beta^{B}\right]_{(r)}
$$
by

The total strain energy of the structural system is given

$$
U=\frac{1}{2}\{q\}^{\prime}[K]\{q\}
$$

and the system kinetic energy by

$$
T=\frac{1}{2}\{\dot{q}\}^{\prime}[M]\{\dot{q}\}
$$

The equations of motion for the system may be derived from Lagrange's equations

$$
\begin{aligned}
&(d / d t)\left(\partial T / \partial \dot{q}_{j}\right)-\left(\partial T / \partial q_{j}\right)+\left(\partial U / \partial q_{j}\right)= \\
& Q_{j} \quad(j=1, \ldots, n)
\end{aligned}
$$

where $Q_{j}$ is the generalized force corresponding to the $j$ th generalized coordinate, exclusive of the force contribution derivable from the elastic strain energy potential $U$. Thus,

$$
[M]\{\ddot{q}\}+[K]\{q\}=\{Q\}
$$

For dynamic response problems it is necessary to determine the time-dependent generalized force vector $\{Q\}$ corresponding to the time-dependent applied forces. This is obtained by examining the virtual work done on the system when virtual displacements $\{\Delta q\}$ are given to the system. The virtual work of the system may be expressed as a sum of the virtual work of the substructures:

$$
\Delta W=\sum_{r}\{P\}_{(r)}{ }^{\prime}\{\Delta p\}_{(r)}=\{P\}^{\prime}\{\Delta p\}=\{Q\}^{\prime}\{\Delta q\}
$$

The last equality requires that the $\{\Delta p\}$ and $\{\Delta q\}$ be consistent, which is guaranteed by Eq. (27). Then, .

$$
\{P\}^{\prime}[\beta]\{\Delta q\}=\{Q\}^{\prime}\{\Delta q\}
$$

and since $\{\Delta q\}$ is completely arbitrary,

$$
\{Q\}=[\beta]^{\prime}\{P\}
$$

Equations (19) and (47) may be combined to give

$$
\{Q\}=[\beta]^{\prime}[\alpha]^{\prime}\{F\}
$$

However, since the substructure force vector $\{F\}$ contains the unknown boundary forces $\left\{F^{B}\right\}$, Eq. (48) does not serve for the initial determination of $\{Q\}$. At this point a set of substructure applied forces will be introduced. In addition, the substructure boundary coordinates $\left\{\delta^{B}\right\}_{(r)}$ must now be separated into $\left\{\delta^{B R}\right\}_{(r)}$ where external loads exist and $\left\{\delta^{B D}\right\}_{(r)}$, which are dummy coordinates that may have been introduced in order to provide for complete boundary constraint. Thus, externally applied forces will exist for the co- 


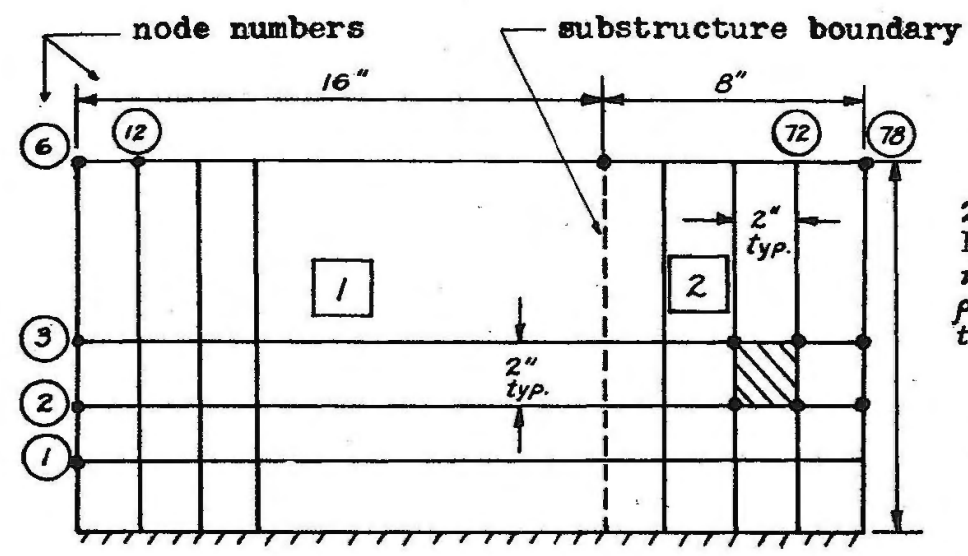

2024-T3 alum. $\mathrm{E}=10.5 \times 10^{6} \mathrm{psi}$. $\nu=0.33$ $\rho \neq 0.101 \mathrm{lb} / \mathrm{in}^{3}$. $t=0.125$ in.
Fig. 3 78-node cantilever rectangular plate. ordinates $\left\{\delta^{I}\right\}_{(r)}$ and $\left\{\delta^{B R}\right\}_{(r)}$. Let $\{\delta\}_{(r)}$ be ordered and partitioned according to

$$
\{\delta\}_{(r)}=\left\{\begin{array}{l}
\frac{\delta^{B D}}{\delta^{B R}} \\
\hdashline \delta^{I}
\end{array}\right\}_{(r)}
$$

and let the set of statically equivalent applied forces be given by

$$
\{\varsubsetneqq\}_{(r)}=\left\{\begin{array}{c}
0 \\
\hdashline \mathfrak{F}^{B R} \\
\hdashline F^{I}
\end{array}\right\}_{(r)}
$$

The generalized forces $\{Q\}$ of the system may be determined from these statically equivalent applied forces by equating the virtual work done by these forces in going through virtual displacements $\{\Delta \delta\}_{(r)}$ and the virtual work done by $\{Q\}$ in going through virtual displacements $\{\Delta q\}$ consistent with $\{\Delta \delta\}$. Thus,

$$
\{Q\}=\sum_{r}\left([ \overline { \beta } ^ { B R } ] _ { ( r ) } \left\{\left\{F^{B R}\right\}+[\beta]_{(r)}^{\prime}\left[\bar{\phi}^{C} \mathrm{i} \bar{\phi}^{N}\right]_{(r)}\left\{\left\{F^{I}\right\}_{(r)}\right)\right.\right.
$$

where $\left[\beta^{B R}\right]_{(r)}$ is the portion of $\left[\beta^{B}\right]_{(r)}$ associated with the retained boundary freedoms $\left\{\delta^{B R}\right\}_{(r)}$.

\section{Free Vibration Example}

In order to check out the procedures outlined in the preceding sections, the free vibration of a cantilever plate was considered. A comparison was made between mode shapes and frequencies of the plate calculated using the stiffness

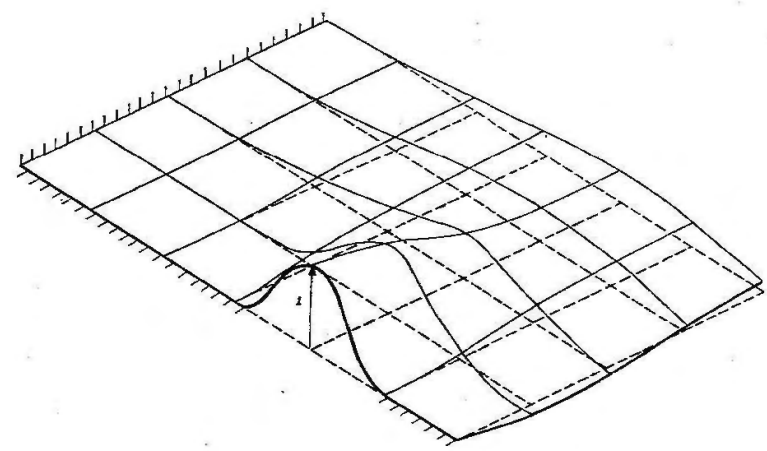

Fig. 4 A constraint mode of plate 2. and mass matrices of a finite-element representation of the full plate with corresponding frequencies and mode shapes calculated by the preceding substructure method. The plate had the dimensions and properties shown in Fig. 3. The substrueture boundary was located so that it would not lie along the line of symmetry of the full plate. To provide for complete restraint along the substructure boundary, rotations have been introduced as "dummy" boundary freedoms. Each sustructure, therefore, has 18 boundary degrees of freedom.

A direct stiffness program was used to generate stiffness matrices for the full plate and for the two substructures. The full-plate stiffness matrix was used in a vibration analysis to obtain modes and frequencies. The substructure stiffness matrices were used as input for the coupling program. The frequencies and mode shapes of the cantilever plate, obtained by using the substructure coupling procedure, were compared with the results obtained using stiffness and mass matrices of the full plate. In both analyses, the mass was lumped at the grid points. No rotatory inertia was included at the dummy boundary rotation freedoms.

Table 1 gives the frequencies obtained by the two methods indicated previously and the amplitudes of several node points for modes 3 and 6 . The coupled-plate solution employed the 18 boundary freedoms plus 5 normal-mode freedoms for each substructure. Figure 4 shows a constraint mode of plate 2, and Fig. 5 gives the shape of a normal mode of the same substructure. Table 2 shows some of the node-point displacements for the sixth mode, which is the third antisymmetric mode of the plate. It may be seen that the coupled-plate solution gives acceptable mode shape results when compared with the full-plate solution. The effect of

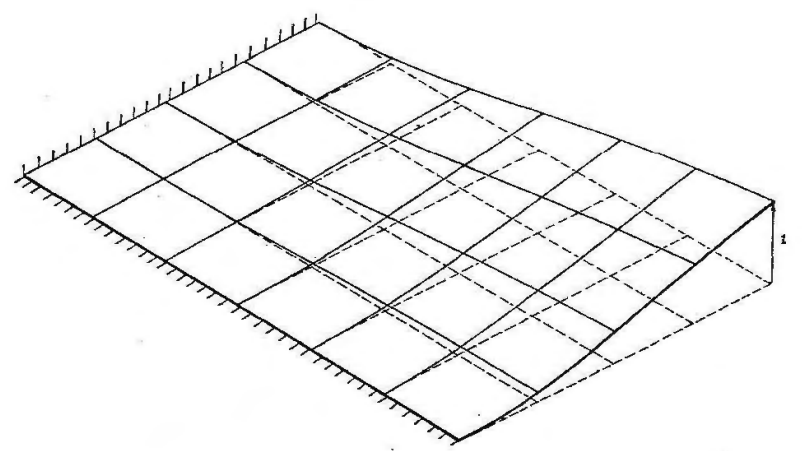

Fig. 5 A normal mode of plate 2 . 
Table 1 Some numerical results for cantilever plate frequencies and mode shapes

\begin{tabular}{|c|c|c|c|c|c|c|c|c|c|c|}
\hline \multirow[b]{2}{*}{ Freq., cps. } & \multicolumn{10}{|c|}{ Mode number } \\
\hline & 1 & 2 & 3 & 4 & 5 & 6 & 7 & 8 & 9 & 10 \\
\hline Full-plate & 29.11 & 44.14 & 83.22 & 152.3 & 174.6 & 198.0 & 247.3 & 272.3 & 343.4 & 400.4 \\
\hline Coupled-plate & 29.13 & 44.17 & 83.31 & 152.8 & 175.1 & 198.3 & 249.1 & 273.6 & 352.8 & 427.9 \\
\hline \multirow[t]{2}{*}{ \% Difference } & 0.06 & 0.05 & 0.10 & 0.28 & 0.27 & 0.16 & 0.69 & 0.45 & 2.15 & 6.87 \\
\hline & & & \multicolumn{7}{|c|}{ Mode number } & \\
\hline \multicolumn{3}{|c|}{ Node no., amplitude } & \multicolumn{2}{|c|}{3 Full } & \multicolumn{2}{|c|}{3 Coupled } & \multicolumn{2}{|l|}{6 Full } & 6 Coupled & \\
\hline \multicolumn{3}{|c|}{6} & \multirow{2}{*}{\multicolumn{2}{|c|}{1.000}} & \multicolumn{2}{|c|}{1,000} & \multicolumn{2}{|l|}{1.000} & 1.000 & \\
\hline \multirow{2}{*}{\multicolumn{3}{|c|}{12}} & & & \multicolumn{2}{|c|}{0.648} & \multicolumn{2}{|l|}{0.821} & 0.804 & \\
\hline & & & \multicolumn{2}{|c|}{0.644} & \multirow{2}{*}{\multicolumn{2}{|c|}{$\begin{array}{r}0.263 \\
-0.122\end{array}$}} & \multicolumn{2}{|l|}{0.570} & 0.542 & \\
\hline \multicolumn{3}{|c|}{24} & \multicolumn{2}{|c|}{-0.121} & & & \multicolumn{2}{|l|}{0.311} & 0.290 & \\
\hline \multicolumn{3}{|c|}{30} & \multicolumn{2}{|c|}{-0.447} & \multicolumn{2}{|c|}{-0.453} & \multicolumn{2}{|l|}{0.117} & 0.113 & \\
\hline \multicolumn{3}{|c|}{36} & \multicolumn{2}{|c|}{-0.668} & \multicolumn{2}{|c|}{-0.672} & \multirow{2}{*}{\multicolumn{2}{|c|}{0.000}} & 0.030 & \\
\hline \multicolumn{3}{|c|}{$42(\notin)$} & \multicolumn{2}{|c|}{-0.746} & \multicolumn{2}{|c|}{-0.744} & & & 0.008 & \\
\hline
\end{tabular}

the deliberate location of the substructure boundary away from the line of symmetry of the plate may be seen by comparing the displacement of symmetrically located node points. For instance, node points 12 and 72 have the same numerical amplitude, 0.8208 , in the full-plate solution, whereas the values 0.8032 and 0.8292 were obtained in the coupled-plate solution. Similar comparisons may be made for other symmetrically located points, and it may be concluded that the accuracy of the mode shapes is not affected adversely by the choice of substructure boundary location. Amplitudes of other modes up to and including the eighth mode indicate that the coupling technique is capable of producing satisfactory mode shapes as well as frequencies. Further results of this investigation are given in Ref. 9 .

Table 2 Mode-shape data, mode 6 (198 cps)

\begin{tabular}{crrrrr}
\hline \hline Node & \multicolumn{1}{c}{ Full } & Coupled & Node $^{a}$ & \multicolumn{1}{l}{ Full } & Coupled \\
\hline 1 & -0.1648 & -0.1645 & 73 & 0.1648 & 0.1661 \\
2 & -0.4708 & -0.4681 & 74 & 0.4708 & 0.4726 \\
3 & -0.5400 & -0.5322 & 75 & 0.5399 & 0.5411 \\
4 & -0.2385 & -0.2269 & 76 & 0.2385 & 0.2381 \\
5 & 0.3450 & 0.3546 & 77 & -0.3450 & -0.3495 \\
$6^{c}$ & 1.0000 & 1.0000 & 78 & -1.0000 & -1.0125 \\
7 & -0.1522 & -0.1505 & 67 & 0.1522 & 0.1550 \\
8 & -0.3967 & -0.3915 & 68 & 0.3967 & 0.4028 \\
9 & -0.4615 & -0.4540 & 69 & 0.4615 & 0.4681 \\
10 & -0.2305 & -0.2252 & 70 & 0.2305 & 0.2345 \\
11 & 0.2416 & 0.2382 & 71 & -0.2416 & -0.2428 \\
12 & 0.8202 & 0.8032 & 72 & -0.8208 & -0.8292 \\
$37^{b}$ & -0.0000 & -0.0026 & & & \\
$38^{b}$ & -0.0000 & -0.0069 & & & \\
$39^{b}$ & -0.0000 & -0.0084 & & & \\
$40^{b}$ & -0.0000 & -0.0057 & & & \\
$41^{b}$ & -0.0000 & 0.0004 & & & \\
$42^{b}$ & -0.0000 & 0.0080 & & & \\
\hline \hline
\end{tabular}

a Nodes in this column are symmetry nodes for points in left-hand column. 6 Nodes on line of symmetry of plate.

$c$ Both solutions normalized on node 6 .

\section{Concluding Remarks}

This paper has described a method of substructure analysis similar to that of Hurty ${ }^{4,5}$ but differing in one important respect; namely, it simplifies the treatment of rigid-body modes of substructures by eliminating the separation of boundary forces into statically determinate and statically indeterminate reactions. This enables all modes associated with boundary freedoms to be treated alike. Furthermore, all such modes may be generated by matrix operations performed by the computer on input substructure matrices. The advantages of the method presented here are that it leads to easier formulation of substructure problems, more compact presentation, simplified computer programming, and, in all likelihood, shorter computer times.

\section{References}

1 Argyris, J. H., "Some Results on the Free-Free Oscillations of Aircraft Type Structures," Revue Francaise Mecan., Vol. 3, 1965, pp. 59-73.

2 Przemieniecki, J. S., "Matrix Structural Analysis of Substructures," AIA A Journal, Vol. 1, No. 1, Jan. 1963, pp. 138-147.

'Taig, I. C., "Automated Stress Analysis Using Substructures," Oct. 1965, paper presented at Conference on Matrix Methods in Structural Mechanies, Wright-Patterson Air Force Base, Ohio.

${ }^{4}$ Hurty, W. C., "Dynamic Analysis of Structural Systems Using Component Modes," AIAA Journal, Vol. 3, No. 4, April 1965, pp. 678-685.

${ }^{5}$ Hurty, W. C., "Dynamic Analysis of Structural Systems by Component Mode Synthesis," Rept. 32-530, 1964, Jet Propulsion Lab., Pasadena, Calif.

${ }^{6}$ Gladwell, G. M. L., "Branch Mode Analysis of Vibrating Systems," Journal of Sound Vibration, Vol. 1, 1964, pp. 41-59.

${ }^{7}$ Hurty, W. C. and Rubinstein, M. F., Dynamics of Structures, Prentice-Hall, Englewood Cliffs, N.J., 1964.

${ }^{8}$ Bisplinghoff, R. L., Ashley, H., and Halfman, R. L., Aeroelasticity, Addison-Wesley, Reading, Mass., 1955.

${ }^{9}$ Craig, R. R. and Bampton, M. C. C., "Coupling of Substructures for Dynamic Analyses," Rept. D6-15509-TN, 1966, The Boeing Company, Commercial Airplane Div., Seattle, Wash. 